Proceedings of the ASME 2012 International Mechanical Engineering Congress \& Exposition

November 9-15, 2012, Houston, Texas, USA

IMECE2012-85236

\title{
EVALUATING MODEL UNCERTAINTY BASED ON PROBABILISTIC ANALYSIS AND COMPONENT OUTPUT UNCERTAINTY DESCRIPTIONS
}

\author{
Magnus Carlsson \\ Saab Aeronautics \\ Linköping, Sweden, SE-581 88 \\ Email: magnus.carlsson@liu.se
}

\author{
Hampus Gavel \\ Saab Aeronautics \\ Linköping, Sweden, SE-581 88
}

\author{
Johan Ölvander \\ Div. of Machine Design \\ Dept. of Management and Engineering \\ Linköping University \\ Linköping, Sweden, SE-581 83
}

\begin{abstract}
To support early model validation, this paper describes a method utilizing information obtained from the common practice component level validation to assess uncertainties on model top level. Initiated in previous research, a generic output uncertainty description component, intended for power-port based simulation models of physical systems, has been implemented in Modelica. A set of model components has been extended with the generic output uncertainty description, and the concept of using component level output uncertainty to assess model top level uncertainty has been applied on a simulation model of a radar liquid cooling system. The focus of this paper is on investigating the applicability of combining the output uncertainty method with probabilistic techniques, not only to provide upper and lower bounds on model uncertainties but also to accompany the uncertainties with estimated probabilities.

It is shown that the method may result in a significant improvement in the conditions for conducting an assessment of model uncertainties. The primary use of the method, in combination with either deterministic or probabilistic techniques, is in the early development phases when system level measurement data are scarce. The method may also be used to point out which model components contribute most to the uncertainty on model top level. Such information can be used to concentrate physical testing activities to areas where it is needed most. In this context, the method supports the concept of Virtual Testing.
\end{abstract}

\section{INTRODUCTION}

Simulation models of physical systems, with or without control software, are widely used in the aeronautic industry, with applications ranging from system development to verification and end-user training. In the effort to reduce the cost of physical testing related to the certification process, the aeronautic industry strives to expand the usage of modeling and simulation (M\&S) further by introducing the concept of Virtual Testing (VT). While no compact and broadly agreed definition of VT has been found, the term VT in this paper refers to the structured use of $M \& S$ to critically evaluate a product's design against specified requirements. In the case of certification, the requirements are set by certification authorities, typically the Federal Aviation Administration in the US or the European Aviation Safety Agency in Europe [1,2] . When VT is used as an Acceptable Means of Compliance in certification, this may be termed Virtual Certification (VC). There is an intuitive analogy between physical testing and VT in terms of the test article and the actual test execution - the test article in physical testing corresponds to a validated simulation model in VT, and the physical test execution corresponds to the simulation in VT. In both cases, it is equally important that test procedures and test setups are well defined.

At the time of writing, EU funded VT related research projects are on-going in all major transportation sectors - from the aeronautic sector to the automotive, railway, and maritime sectors. One example from the aeronautic sector is the CRESCENDO project, in which methodologies and tools 
intended to enable collaborative design, VT, and VC are being developed [3]. It should be emphasized that the CRESCENDO VT and VC approaches are intended to support the current certification process, and that VT will not replace physical testing. Instead, VT is intended to be the means to better plan physical testing, to reduce the number of physical tests, and to reduce risk associated with physical testing.

The importance of Verification and Validation $(V \& V)$ of simulation models is well known and the V\&V research field has a long history, see for example Naylor and Finger [4] who propose a method named multi-stage verification, and Sargent [5] who provides an overview of the subject and describes a set of validation techniques. In today's developments of VT towards VC, the challenging task of assessing a model's validity is nonetheless of greater importance than ever. In a broader perspective, model validation is only one factor in the assessment of the credibility of a M\&S activity. For examples of credibility assessment methods, see the Credibility Assessment Scale proposed in the NASA Standard for Models and Simulations [6], the Predictive Capability Maturity Model proposed by Sandia National Laboratories [7], and the Validation Process Maturity Model proposed by Harmon and Youngblood [8]. A brief summary of these three methods is provided by Carlsson et al. [9].

With the above credibility scope in mind, this paper zooms into model validation, and more specifically into early model validation, which here refers to assessment of a model's validity in lack of system level measurement data. A main research question is: Is there an industrial applicable way to use information on component level uncertainty to draw conclusions on model top level uncertainty? As an answer, this paper proposes a pragmatic approach to how to utilize uncertainty information obtained from the common practice of component validation to assess uncertainties on model top level. Previous research has shown that the method may result in a significant reduction of the number of uncertain parameters that require consideration in a simulation model, and the method has been tested in combination with a set of deterministic techniques [10]. When the number of uncertain parameters to take into account has been successfully reduced, probabilistic techniques may be considered even for computationally expensive models. The method is primarily intended for large scale mathematical 1-D dynamic simulation models of physical systems with or without control software, typically described by Ordinary Differential Equations (ODE) or Differential Algebraic Equations (DAE).

The following section introduces the reader to early model validation and provides the context of the proposed method. The proposed method is then combined with probabilistic techniques and applied in an uncertainty analysis of a simulation model of a radar liquid cooling system. The final section contains conclusions and recommendations to consider when applying the proposed method in uncertainty analysis of simulation models.

\section{EARLY MODEL VALIDATION}

Several definitions of the terms verification and validation exist, some of them collected in the Generic Methodology for Verification and Validation (GM-VV) [11]. As formulated by Balci [12], verification concerns building the model right, i.e. determining whether the model is compliant with the model specification and if it accurately represents the underlying mathematical model. Validation concerns building the right model, i.e. determining whether the model is a sufficiently accurate representation of the real system of interest from the perspective of the intended use of the model. This brief description of $\mathrm{V} \& \mathrm{~V}$ terminology is in line with definitions used by NASA [6], ITOP [13], and the US DoD [14].

Balci [12] lists more than 75 techniques for verification, validation, and testing (VV\&T), divided into four groups; informal, formal, static, and dynamic. These are further described in Balci [15]. Another well-established set of validation techniques is provided by Sargent, see Ref. [16] for an up-to-date version. As indicated above, Sargent's list concerns validation techniques only, while Balci's list contains a mix of VV\&T techniques, and it is not always easy to determine whether a specific technique should be considered to be directed towards verification or validation. It is the authors' understanding that informal techniques like face validation and reviews are generic and may concern both verification and validation. Informal techniques are of great importance and often easy to apply, but will not be further discussed in this paper. Formal techniques based on mathematical proof of correctness may also cover both verification and validation aspects. However, as indicated by Balci [15], formal methods are rarely applicable where complex simulation models are cencerned. Static techniques like interface analysis and structural analysis are believed to be directed more towards verification than validation. Left are the group of dynamic techniques which, as clarified in the sections below, are of most interest to this paper.

$\mathrm{V} \& \mathrm{~V}$ of simulation models is sometimes seen as activities carried out at the end of the modeling process - in particular the validation activity which may require a large amount of measurement data from the real system of interest. When using M\&S to take early model-based design decisions - when no physical prototype of the system exists - it is still important to assess the uncertainty in the simulation results. In addition to this, the authors experience from M\&S of aircraft vehicle systems is that there tend to be a persistent lack of system level measurement data for validation purposes, also in the later development stages. In practice, when modeling for example an aircraft subsystem, one never has access to system level measurement data covering all points in the flight envelope. To what extent may the results from the validation against measurement data then be interpolated/extrapolated? Since this question may be hard to answer, it is important to be able to assess model uncertainties with only limited system level measurement data available. Such an assessment would constitute an important part of early model validation. 
With the purpose of facilitating early model validation, this paper proposes a method based mainly on a combination of the dynamic techniques denoted by Balci as sub-model/module testing, bottom-up testing, and predictive validation. As described in the following sections, the proposed method may be combined with sensitivity analysis and/or optimization techniques, and applied in deterministic- as well as probabilistic frameworks to enable simulation model uncertainty analysis.

Uncertainty analysis in this paper refers to the process of identifying, quantifying, and assessing the impact of uncertainty sources embedded along the development and usage of simulation models. A few examples of potential sources of uncertainty are model parameters, model boundary conditions, model simplifications, and the numerical method used by the solver. According to Roy and Oberkampf [17], all uncertainties originate from three key sources; model inputs, numerical approximations, and model form uncertainty. This is in line with the definitions provided by Coleman and Steele [18]. Commonly, a distinction is made between aleatory uncertainty (due to statistical variations, also referred to as variability, inherent uncertainty, irreducible uncertainty, or stochastic uncertainty) and epistemic uncertainty (due to lack of information, also referred to as reducible uncertainty or subjective uncertainty). See Padulo [19] for an extensive literature review of uncertainty taxonomies.

\section{THE OUTPUT UNCERTAINTY METHOD}

To help the reader understand the proposed method, a simulation model of a radar liquid cooling system is used as an industrial application example. The method was originally described by Carlsson et al. [10] by the means of a scenario description. The following sub-sections introduce the industrial application example and describe the method using a short version of the scenario.

\section{Industrial Application Example}

A simulation model of the radar liquid cooling system in a Saab Gripen Demonstrator Aircraft is used as an illustrative example. The model was developed in the Modelica based M\&S tool Dymola $[20,21]$. The main components in the system are pump, accumulator, liquid-to-air heat exchanger, piping, and a subsystem of heat loads including the radar antenna and related electronic equipment. The simulation model layout is shown in the picture below, which also includes information to distinguish between components and sub-models. In the figure below, a component is a model of a single piece of equipment and a sub-model includes several components.

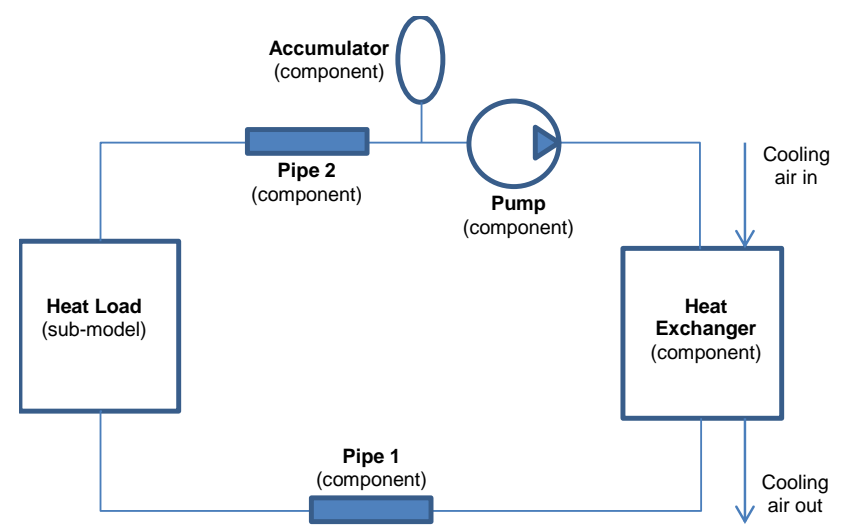

Figure 1: LAYOUT OF THE RADAR LIQUID COOLING SYSTEM.

From a system simulation perspective, this model may appear fairly simple. Yet it is a component based model of a physical system, including a number of components and one sub-model. This 1-D dynamic simulation model is used to predict pressure, mass flow, and temperature levels at different points in the system. The components include equations describing pressure variations due to g-loads and fluid thermal expansion, internal heat exchange between equipment and fluid, external heat exchange between equipment and surrounding equipment bays, temperature dynamics in equipment and fluid, as well as fluid dynamics due to transport delays in the piping arrangement. The model includes approximately 200 equations, 100 parameters, and 50 states. The radar liquid loop model was developed using a subpackage of a component library developed at Saab Aeronautics and uses a connector interface that includes information about pressure, mass flow, and specific enthalpy $(p, \dot{m}, h)$.

\section{Motivation of Method}

Prior to initiating the development of a simulation model's components and sub-models, there are normally activities such as specifying the intended use of the model, deriving model requirements, defining model layout and interfaces, and producing a $\mathrm{V} \& \mathrm{~V}$ plan [9]. In the following short scenario, these initial activities are assumed to be completed and we move straight on to what one may call the core of model development. Briefly described, a typical approach in component based modeling is to a) model each component or if possible select suitable components from a component library, b) perform $V \& V$ activities on component level, which is often an iterative process including tuning of component parameters, and c) assemble sub-models up to model top level.

Available information on component level typically used in steps a) and b) may for example be datasheets, rig test data for similar components, or component level CFD simulation results. Thus, after carrying out the component $\mathrm{V} \& \mathrm{~V}$ activities in step b), there is indeed uncertainty information available for the individual components and sub-models. However, in the authors' experience this uncertainty information on component level is not always utilized at model top level. To summarize the problem - uncertainties of the components are known to 
some degree, but what is the uncertainty on model top level? For example, what is the uncertainty in the pressure at the heat load input port in the liquid cooling model? Reasonably, it should be possible to utilize our knowledge of the uncertainties on component level and sub-model level to estimate the uncertainties on top level.

Where system level measurement data is unavailable, a common approach is to perform a sensitivity analysis, e.g. by varying component parameters and performing a simulation for each parameter change to determine how different parameters affect the model output. However, in the scenario described above we have knowledge of the uncertainties of the component characteristics (output), but we do not know the uncertainties in the component parameters (input). Due to lack of information on parameter uncertainty, quantifying uncertainties in component parameters is often a difficult task. As an example - what is a suitable range of the roughness coefficient in component "Pipe 1", or what does the probability density function look like? Quantifying parameter uncertainties in models with many parameters is thus not always feasible.

From an uncertainty analysis point of view there is a drawback if the only thing that is varied in the sensitivity analysis is a model's original component parameters - the uncertainties in a model's original component parameters only cover one aspect of the total model uncertainty. In that case, other kinds of uncertainties, like uncertainties of underlying equations or uncertainties due to model simplifications, are ignored.

In addition to this, sensitivity analysis applied on models with many parameters requires a large number of simulations. One approach to mitigate the computational heaviness of the sensitivity analysis is to use simplified models, also known as meta-models or surrogate models, e.g. response surfaces of varying order [22]. By definition there is a discrepancy between the surrogate model and the original model of interest. In this approach additional $\mathrm{V} \& \mathrm{~V}$ tasks therefore need to be performed. If a sensitivity analysis is carried out on the surrogate model, knowledge is gained of how the parameters affect the surrogate model output and not the output of the original model.

\section{Description of Method}

To answer the question "What is the uncertainty on model top level?", given the constraints regarding large scale physical models as well as the lack of system level measurement data, this section proposes an approach based on the original model components extended with an uncertainty description utilizing available information on component output uncertainty. As the model components may be legacy code or originate from a Commercial Off The Shelf (COTS) component library, it is favorable to keep them unmodified. Andersson [23] describes how a fault injection block may be implemented in signal flow models. At Saab Aeronautics, this kind of fault injection feature has proven to be useful for simulation of different kind of faults in mid-scale and large-scale simulators, for example sensor failures of various kinds. The method proposed in this paper is similar to the fault injection feature for signal flow models, except that consideration must be given to the power port concept commonly used in physical modeling.

The idea is to develop a new uncertain component by including an original component and adding an uncertainty description component. The uncertainties are introduced in the uncertainty description component by including equations for modifying one or more of the variables in the connector interface. The uncertainties may be expressed in absolute terms or relative to some characteristic of the original component. As this approach enables uncertainties to be defined for a component's outputs rather than its inputs, the method is termed output uncertainty. A brief description is given below of how the method is implemented in the thermal-fluid component library used in the liquid cooling model. For equations and further implementation aspects, see Ref. [10].

In the component library used for the liquid cooling model, the connector interface includes information on pressure, mass flow, and specific enthalpy $(p, \dot{m}, h)$. In the aim to achieve an intuitive uncertainty description, it has been chosen to add uncertainties in terms of pressure and temperature (the latter implicitly meaning specific enthalpy). This is appropriate since pressure and temperature are two commonly used entities when measuring or specifying system characteristics. In line with the discussion above, two types of uncertainty descriptions have been implemented - absolute and relative. The absolute uncertainty component introduces two parameters; pressure uncertainty $p_{U C}[\mathrm{~Pa}]$ and temperature uncertainty $T_{U C}[\mathrm{~K}]$. The relative uncertainty component uses similar parameters, but relative to the pressure difference and temperature difference over the original component; relative pressure uncertainty $p_{R U C}$ [-] and relative temperature uncertainty $T_{R U C}[-]$.

It should be noted that - as when varying for example a component's pressure loss coefficient - varying a component's pressure uncertainty parameter corresponds to a variation of the component's pressure drop characteristics. Thus, introducing uncertainties in pressure implies uncertainties in mass flow. The figure below shows an example of the pressure drop characteristics of a pipe component with absolute and relative uncertainty respectively.

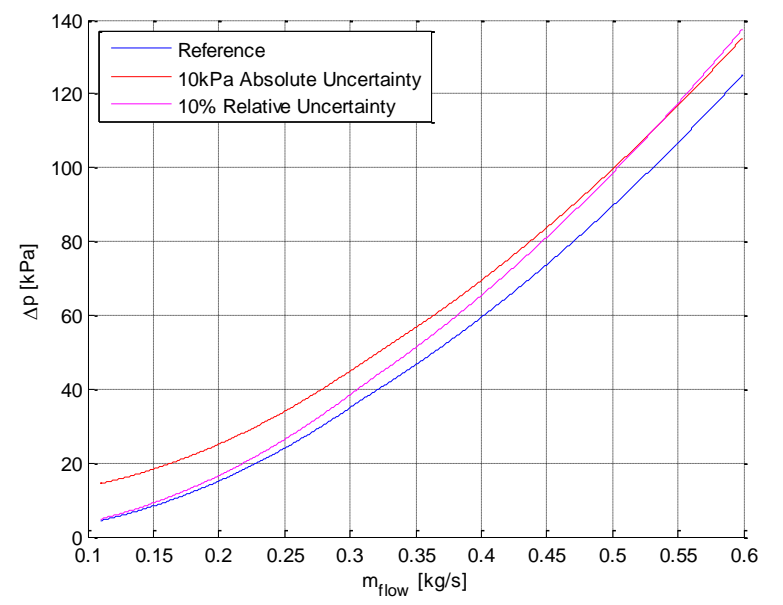

Figure 2: ABSOLUTE UNCERTAINTY VERSUS RELATIVE UNCERTAINTY. 
Based on existing components, a new component library with uncertainty descriptions is created. As an example, a new component UncertainPipe is created by including an original pipe component and a relative uncertainty component, and propagating all parameters to component top level. From a user point of view, the UncertainPipe looks like the original pipe component with the two additional parameters $p_{R U C}$ and $T_{R U C}$. Note that this is done for all flow type components in the model (pump, HEX, pipe1, AESA, and pipe2). The figure below shows the liquid cooling model updated with the appropriate uncertain components, as well as how the uncertainty description components are connected with the original components.

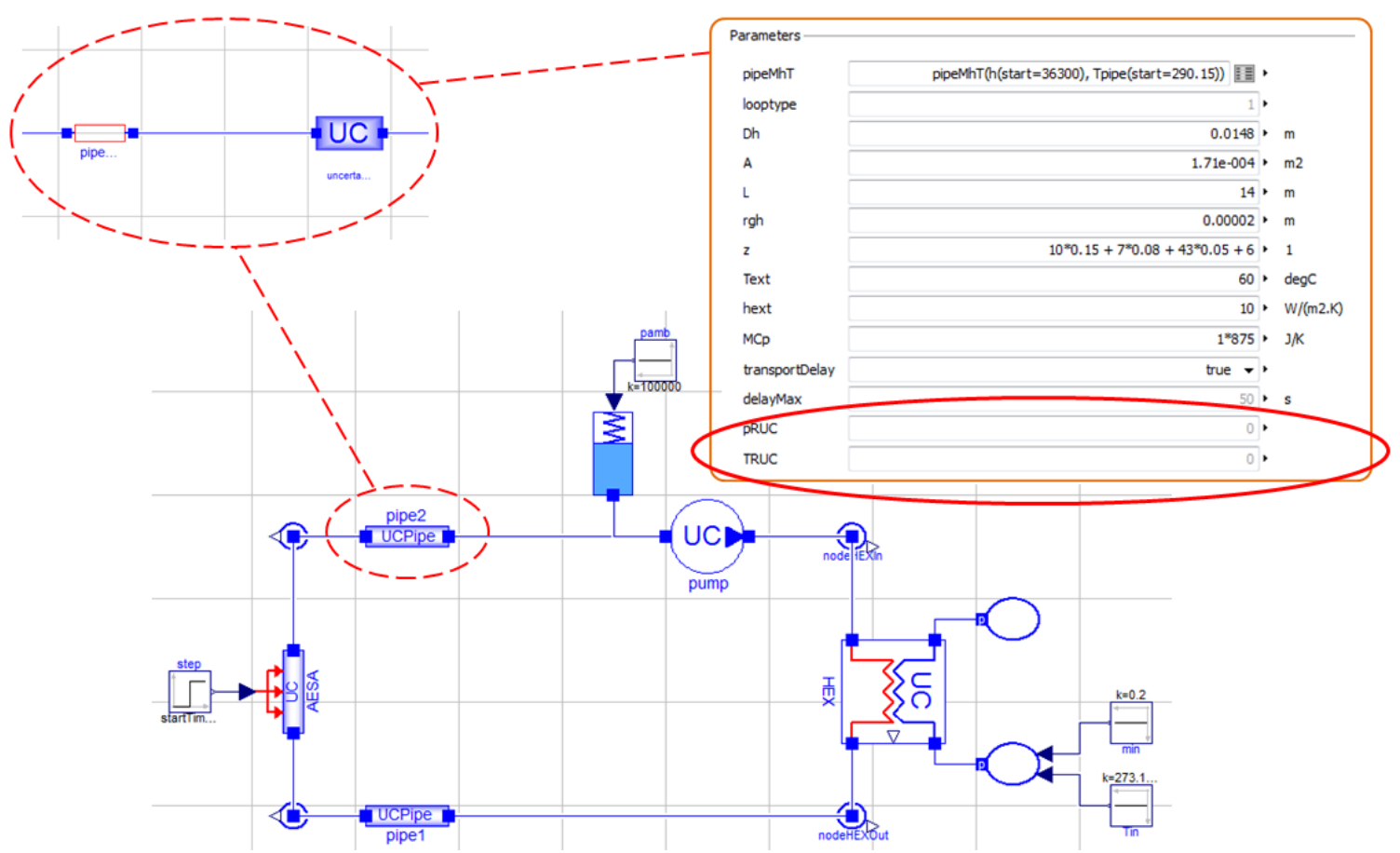

Figure 3: RADAR LIQUID COOLING MODEL, UPDATED WITH COMPONENTS INCLUDING AN OUTPUT UNCERTAINTY DESCRIPTION. THE TWO NEW PARAMETERS IN THE PARAMETER DIALOG ARE MARKED WITH A RED ELLIPSE.

\section{Context of Method}

To define the context of the output uncertainty method and to clarify the difference compared to alternative methods, Figure 4 is provided. The figure aims to visualize that uncertainty analysis of simulation models may be carried out in several different ways, by combining a set of techniques. The figure does not claim to show all possible ways of performing an uncertainty analysis, but is intended to show alternatives closely related to the proposed output uncertainty method. As indicated in the figure, one approach to assess simulation model uncertainties is to use the nominal (or "original") model in combination with some deterministic or probabilistic technique.
In the case of sensitivity analysis (SA), simply using upper and lower bounds on parameter values would imply a deterministic uncertainty analysis, while using probability density functions would imply a probabilistic uncertainty analysis.

Starting from the top of the figure and following the arrows down to the bottom, a set of different tool chains are obtained. Naturally, each tool chain has its own benefits and drawbacks regarding for example execution time, management effort, availability of uncertainty information, and results information content. However, assessing the benefits and drawbacks of each alternative tool chain is beyond the scope of this paper. 


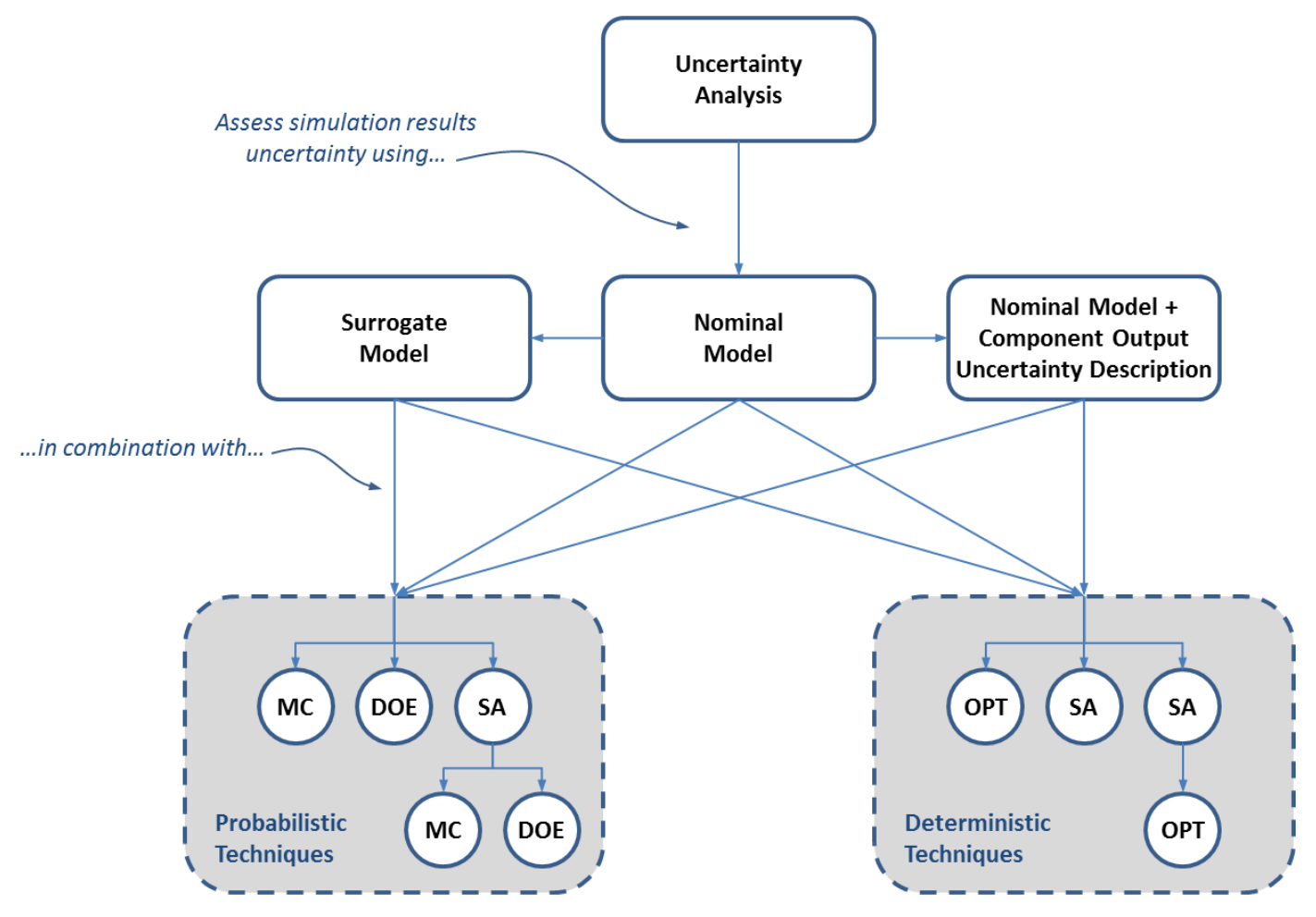

OPT: Optimization (e.g. find maximum/minimum of system characteristics) SA: Sensitivity Analysis (local or global)

MC: Monte Carlo Sampling

DOE: Design Of Experiments (e.g. Latin Hypercube Sampling)

Figure 4: ALTERNATIVE APPROACHES FOR ASSESSMENT OF SIMULATION MODEL UNCERTAINTY.

For an example partly exploring the left part of the figure, see Persson and Ölvander [24] who compare sampling techniques using a simulation model of a dynamic pressure regulator as an application example. The following tool chains are discussed by Persson and Ölvander, see Figure 4 for definitions of abbreviations:

- $\quad$ Nominal Model $\rightarrow$ Probabilistic Techniques $\rightarrow \mathrm{SA} \rightarrow$ $\mathrm{MC}$

- $\quad$ Nominal Model $\rightarrow$ Probabilistic Techniques $\rightarrow \mathrm{SA} \rightarrow$ DOE

- Nominal Model $\rightarrow$ Surrogate Model $\rightarrow$ Probabilistic Techniques $\rightarrow \mathrm{SA} \rightarrow \mathrm{MC}$

- $\quad$ Nominal Model $\rightarrow$ Surrogate Model $\rightarrow$ Probabilistic Techniques $\rightarrow \mathrm{SA} \rightarrow \mathrm{DOE}$

To clarify, the first of the above tool chains refers to using the nominal model in a probabilistic analysis of model uncertainties by means of an initial sensitivity analysis to locate the most significant parameters. These parameters are then assigned probability density functions, and Monte Carlo sampling is used to find the output distributions. In the second of the above tool chains, the Monte Carlo sampling is replaced by a more efficient sampling technique, in this case Latin Hypercube sampling. The two last of the above tool chains are similar to the first two, with the exception that the nominal model is replaced by a surrogate model. In these two cases, the surrogate model is introduced with the aim of reducing execution time. For discussions on the right part of the figure, see Carlsson et al. [10] who use the radar liquid cooling model to study the following tool chains:

- Nominal Model $\rightarrow$ Output Uncertainty $\rightarrow$ Deterministic Techniques $\rightarrow$ OPT

- Nominal Model $\rightarrow$ Output Uncertainty $\rightarrow$ Deterministic Techniques $\rightarrow$ SA

- Nominal Model $\rightarrow$ Output Uncertainty $\rightarrow$ Deterministic Techniques $\rightarrow \mathrm{SA} \rightarrow \mathrm{OPT}$

Common to all three of the above tool chains are that the nominal model is extended with component output uncertainty descriptions. In the first of the above cases, the output uncertainty parameters are used as design variables, and optimization is used to find the minimum and maximum values for a set of selected system characteristics. In the second, a quantitative sensitivity analysis is used to find the minimum and maximum system characteristics. The third case is a combination using an initial sensitivity analysis to locate the most significant parameters. This reduced set of parameters is 
then used as design variables in the optimization to find the minimum and maximum system characteristics.

In the next section, the output uncertainty method is combined with probabilistic techniques as listed below:

- Nominal Model $\rightarrow$ Output Uncertainty $\rightarrow$ Probabilistic Techniques $\rightarrow$ MC

- Nominal Model $\rightarrow$ Output Uncertainty $\rightarrow$ Probabilistic Techniques $\rightarrow$ DOE

In both cases, the nominal model is extended with component output uncertainty descriptions. Available information from the component validation is utilized to assign probability density functions to the output uncertainty parameters, and two different sampling techniques (Monte Carlo and Latin Hypercube respectively) are then used to find the output distributions.

\section{PROBABILISTIC UNCERTAINTY ASSESSMENT}

\section{Analysis Setup}

To study the applicability of the output uncertainty method in combination with probabilistic techniques, the model of the liquid cooling system is updated with component output uncertainty descriptions according to Figure 3. The system characteristics in the five node points of the model are considered to be of general interest. Of special interest in this analysis are the pressure and temperature levels at the heat load input port. The boundary conditions are defined by a static flight case and the radar heat load is modeled as a step change.

The nominal (or "original") liquid cooling model has approximately 100 parameters, but only a subset of 22 parameters are considered uncertain and thereby of interest for the study. These are component parameters affecting pressure and temperature levels, such as pressure loss coefficients, pipe roughness coefficients, and heat transfer coefficients. Many of the parameters that are out of scope for the uncertainty analysis are geometry parameters considered to be known. Model inputs for specifying load case or "simulation scenario" are also treated deterministically. Examples of such model inputs are boundary conditions for definition of flight case (e.g. Mach number, altitude, g-loads, and atmosphere model) and input signals representing cooling air mass flow, cooling air temperature, and heat load power. Using the output uncertainty method, the number of parameters that require consideration is reduced from 22 to 10 . This number originates from the fact that the model includes five flow type components (pump, $H E X$, pipe 1, AESA, and pipe2), with two additional parameters each $\left(p_{R U C}, T_{R U C}\right)$. To clarify, all original parameters are kept with nominal values, but the model has been extended with 10 new parameters. These 10 parameters are considered uncertain, and are assigned probability density functions as described below.

Component validation was performed mainly by comparing simulated component characteristics with component level rig test data and performance figures obtained from datasheets and specifications. Based on the information available from the component validation, the 10 output uncertainty parameters are assigned symmetric uniform distributions with zero mean (an output uncertainty parameter value of zero implies nominal component characteristics). In this analysis, the uncertainties are mainly due to lack of information and are thereby considered epistemic. An assessment using different solvers and varying tolerances indicates that the numerical error in this type of simulation is insignificant compared to other simulation result uncertainties. In this analysis, the simulation result uncertainties due to numerical approximations are therefore ignored.

The analysis include a basic comparison of a brute force Monte Carlo sampling versus a Latin Hypercube Sampling (LHS). The liquid cooling model, which is compiled in Dymola, is called from a MATLAB-script managing preprocessing, sampling, and post processing.

\section{Results}

A basic result is that the liquid cooling model extended with component uncertainty descriptions simulates and, for the verified test cases, behaves as intended. Also, the above discussed probabilistic tool chain runs and provides reasonable results.

As discussed under Analysis Setup in the previous section, one main result of extending the nominal model with component output uncertainty descriptions is that the number of parameters that requires consideration in the uncertainty analysis can be reduced from 22 to 10 . Moreover, the uncertainty quantification of this new reduced set of parameters comes to be more intuitive, since information obtained from the component $\mathrm{V} \& \mathrm{~V}$ activities can be utilized.

If the study had been carried out in the form of a full factorial experiment and each of the 10 input parameters had been divided into say 10 discrete numbers, this would require a number of $10^{10}$ simulations. Since each simulation run of a simple 500-second flight case takes about 2.1 seconds (on a standard PC with an Intel ${ }^{\circledR}$ Core $^{\mathrm{TM}}$ i5 CPU), this was not an option. Instead, the strategy used is to perform a "sufficiently high" number of Monte Carlo samples, and use the results obtained for comparisons. To determine what is a "sufficiently high" number of samples, one approach is to evaluate the convergence of the mean and standard deviation of the simulation results. The following figures show the mean of the maximum pressure and temperature at the heat load input port, for both of the sampling methods used. 

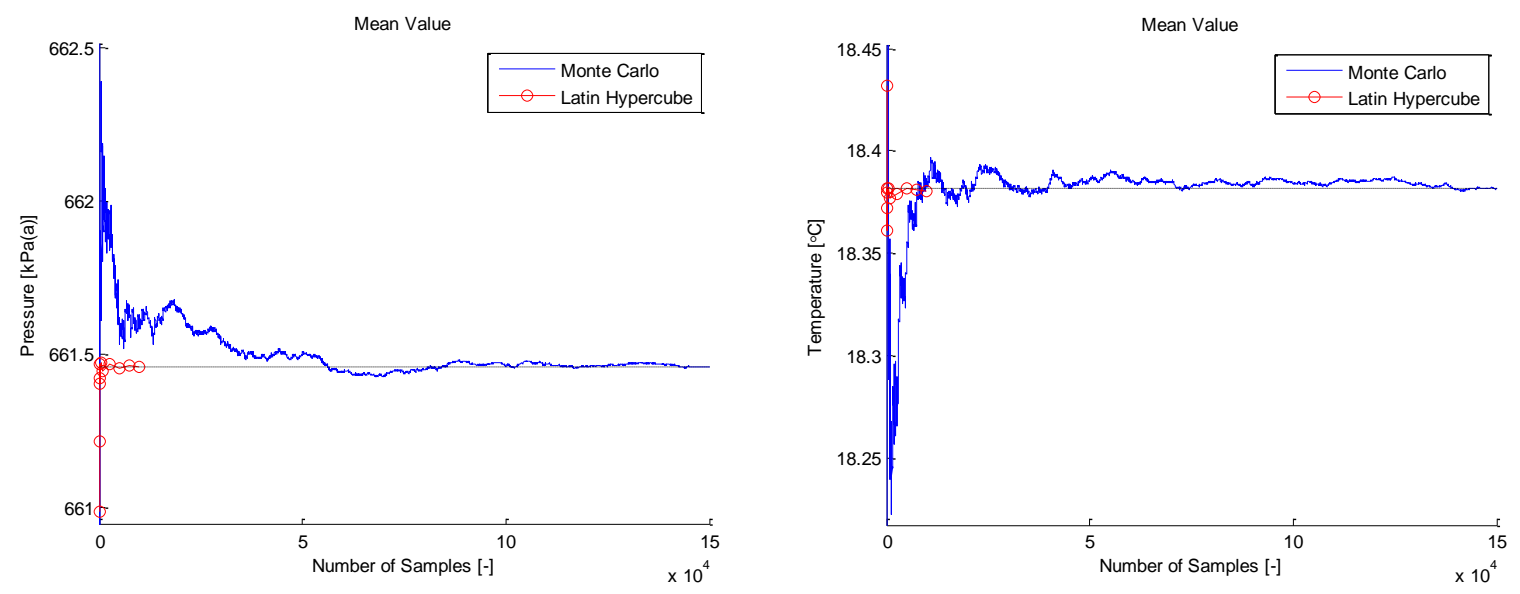

Figure 5: MEAN VALUE COMPARISON FOR HEAT LOAD INLET PRESSURE AND TEMPERATURE RESPECTIVELY.

An evaluation of the simulation results by studying mean values shows that Monte Carlo sampling requires a significantly higher number of samples to converge compared to LHS. This result applies to the system characteristics at all five node points throughout the model. Naturally, what is considered an acceptable convergence limit may differ between applications. For the convergence of standard deviations, a less clear result is obtained.

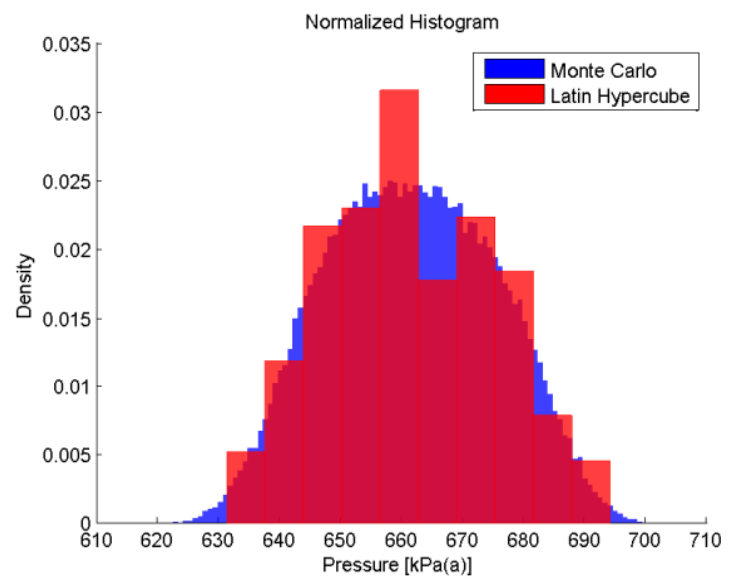

It is also interesting to study how well the distributions obtained with LHS correspond to the distributions obtained with Monte Carlo sampling. The following figures show the distributions of the pressure and temperature at the heat load input port, for Monte Carlo sampling with $1.5 \cdot 10^{5}$ samples and LHS with 250 intervals. It can be noted that the shape of the distributions is preserved fairly well even for this low number of LHS intervals.

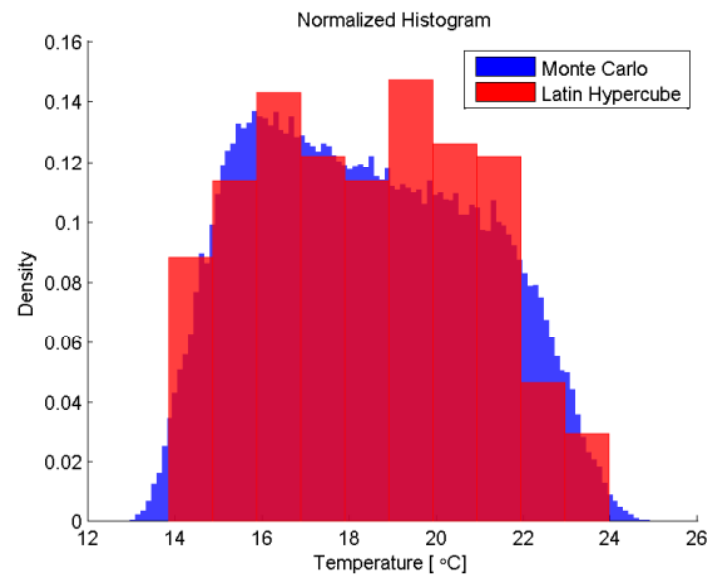

Figure 6: OUTPUT DISTRIBUTIONS OF HEAT LOAD INLET PRESSURE AND TEMPERATURE RESPECTIVELY.

Probability distributions of system characteristics, like those shown in the figure above, constitute useful information in the assessment of model top level uncertainties - in particular when system level measurement data are scarce. In early model validation, it is interesting to evaluate the range of the system characteristics with respect to the intended use of the model. If the range is deemed too large, a feasible approach is to use sensitivity analysis to point out which components contribute most to the uncertainty on model top level, and if possible try to decrease the uncertainty of those components. However, it is important not to confuse variations of system characteristics due to flight cases with variations due to component uncertainties. A sensitivity analysis using the limits of the 10 output uncertainty parameters shows that the pressure output uncertainty of the components pump, pipel, and AESA are the three major contributors to the uncertainty on model top level.

The liquid cooling model is intended to be used separately, as a standalone model to facilitate designing and specifying the liquid cooling system. Obviously, when specifying for example the burst pressure for the radar antenna, it is important to have an understanding not only of the nominal pressure levels at the antenna inlet port but also of the uncertainties. A useful visualization alternative in such cases is the cumulative distribution, which provides the probability of a system characteristic being less than or equal to a specific value. 

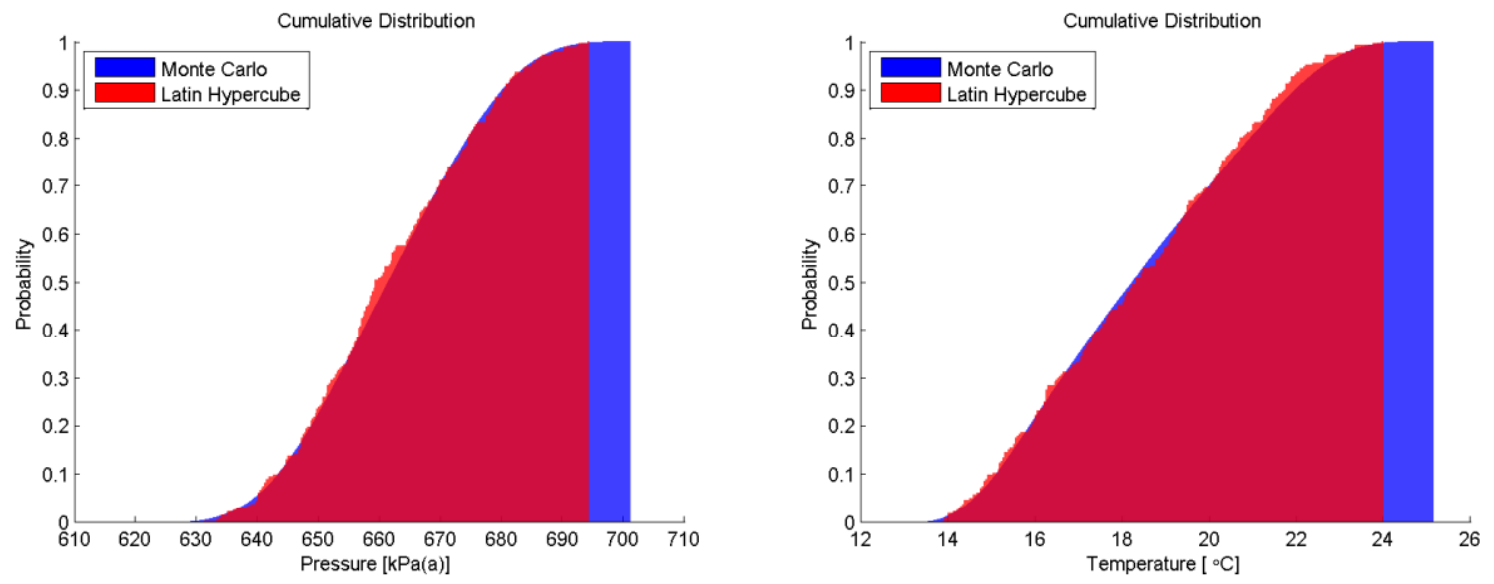

Figure 7: CUMULATIVE DISTRIBUTIONS OF HEAT LOAD INLET PRESSURE AND TEMPERATURE RESPECTIVELY.

As another example of intended use, the liquid cooling model may be integrated in a simulator environment in which it is connected to other simulation models, such as the radar model. A simulator may include models of different fidelity, and different models typically have different requirements regarding input accuracy. Assessing uncertainties at simulator level is indeed a difficult task but distributions of system characteristics for each model integrated in the simulator would be a good starting point.

\section{DISCUSSION AND CONCLUSIONS}

This paper proposes a method of utilizing information obtained from the common practice of component validation to assess uncertainties on model top level. Focusing on industrial applicability, the method makes use of information normally available to engineers developing simulation models of existing or not yet existing systems. As this approach enables defining uncertainties for a component's outputs (characteristics) rather than its inputs (parameters), this method is here termed output uncertainty.

The primary use of the output uncertainty method, in combination with either deterministic or probabilistic techniques, is in the early development phases when system level measurement data are scarce. One example is when designing a system and specifying its components. Compared to specification of system components based on nominal simulation results only, having prior knowledge of model top level uncertainties would decrease the risk of specification errors. Another benefit of the output uncertainty method, which is more related to Virtual Testing, is the ability to show which model components contribute most to the uncertainty on model top level. Such information may be used to better plan physical testing, i.e. to concentrate physical testing activities to areas where it is needed most. In this context, the output uncertainty method to some extent contributes to the aeronautic industry's effort to reduce the cost of physical testing.

In the Results section, it is shown that - compared to an uncertainty analysis using a model's original component parameters - the method may result in a significant reduction of the number of uncertain parameters that require consideration in a simulation model. In the industrial application example used, the number of uncertain parameters that require consideration is reduced by more than $50 \%$. In combination with the more intuitive uncertainty quantification of these parameters, this implies a substantial improvement in the conditions for conducting an assessment of model uncertainties. The method has earlier been used in combination with deterministic techniques to estimate minimum and maximum values of selected system characteristics, see Carlsson et al. [10].

If the number of uncertain parameters that require consideration can be sufficiently reduced and the simulation model and flight cases of interest do not imply too long execution time, a probabilistic uncertainty analysis may be feasible. On the one hand, compared to the deterministic uncertainty analysis, the probabilistic uncertainty analysis is more demanding, but on the other hand it gives more in return. It is more demanding in terms of both execution time and availability and preparation of input data. It gives more in return since the resulting system characteristics are expressed as probability distributions. This is an obvious difference compared to the deterministic uncertainty analysis, which does not provide any information on the probabilities of the resulting minimum and maximum values.

For the liquid cooling model in combination with the used flight case, the system characteristics obtained with a low number of Latin Hypercube samples (250 samples) are in fairly good agreement with the system characteristics obtained with a high number of Monte Carlo samples (1.5.10 5 samples). With the above benefits and drawbacks in mind, the output uncertainty method in combination with efficient sampling techniques actually makes a probabilistic uncertainty analysis feasible for this type of application. As always, the credibility of such an analysis depends on the credibility of the input data. 


\section{ACKNOWLEDGEMENTS}

The research leading to these results has received funding from the European Union Seventh Framework Programme (FP7/2007-2013) under grant agreement $\mathrm{n}^{\circ} 234344$ (www.crescendo-fp7.eu/). We also wish to thank the Swedish Governmental Agency VINNOVA's National Aviation Engineering Research Programme (NFFP5 2010-01262) and Saab Aeronautics for sponsoring this work. Thanks are also due to Sören Steinkellner at Saab Aeronautics for his comments on drafts of this paper.

\section{REFERENCES}

[1] FAA 2012. Federal Aviation Administration. Accessed February 23. http://www.faa.gov/.

[2] EASA 2012. European Aviation Safety Agency. Accessed February 23. http://easa.europa.eu/.

[3] CRESCENDO 2012. Collaborative \& Robust Engineering using Simulation Capability Enabling Next Design Optimisation. Seventh Framework Programme (FP7). Project Reference 234344. http://www.crescendo-fp7.eu/.

[4] Naylor, T. H. and J. M. Finger. 1967. "Verification of computer simulation models". Management Science, 14, 2: B92-B101.

[5] Sargent, R. G. 1979. "Validation of Simulation Models". In Proceedings of the 1979 Winter Simulation Conference, Edited by H. J. Highland, M. G. Spiegel, and R. Shannon: 497-503.

[6] NASA 2008. "Standard for Models and Simulations". NASA-STD-7009. National Aeronautics and Space Administration, Washington, DC 20546-0001

[7] Oberkampf, W. L., Pilch, M., Trucano, T. G., 2007. "Predictive Capability Maturity Model for Computational Modeling and Simulation". SAND2007-5948. Sandia National Laboratories, Albuquerque, New Mexico 87185 and Livermore, California 94550

[8] Harmon, S. Y., Youngblood, S. M., 2005. "A Proposed Model for Simulation Validation Process Maturity", The Journal of Defense Modeling and Simulation, 2, 4: 179-190.

[9] Carlsson, M., Andersson, H., Gavel, H., Ölvander, J., 2012a. "Methodology for Development and Validation of Multipurpose Simulation Models", 50 ${ }^{\text {th }}$ AIAA Aerospace Sciences Meeting, Nashville, Tennessee

[10] Carlsson, M., Steinkellner, S., Gavel, H., Ölvander, J., 2012b. "Utilizing Uncertainty Information in Early Model Validation", AIAA Modeling and Simulation Technologies Conference, Minneapolis, Minnesota

[11] GM-VV 2010. "Generic Methodology for Verification and Validation (GM-VV) to Support Acceptance of Models, Simulations, and Data, Reference Manual", SISO-GUIDE00X.1-201X-DRAFT-V1.2.3
[12] Balci, O. 1997. "Verification, Validation and Accreditation of Simulation Models". In Proceedings of the 1997 Winter Simulation Conference, Edited by S. Andradóttir, K. J. Healy, D. H. Withers, and B. L. Nelson: 135-141.

[13] ITOP 2004. General Procedure for Modeling and Simulation Verification \& Validation Information Exchange, International Test Operations Procedure, ITOP 1-1-002

[14] US DoD 2007. US Department of Defense Directive, Number 5000.59

[15] Balci, O. 1998. Verification, validation, and testing. In The Handbook of Simulation, Chapter 10, J. Banks, Ed., John Wiley \& Sons, New York, NY

[16] Sargent, R. G. 2010. "Verification and Validation of Simulation Models". In Proceedings of the 2010 Winter Simulation Conference, Edited by B. Johansson, S. Jain, J. Montoya-Torres, J. Hugan, and E. Yücesan: 166-183.

[17] Roy, C. J., Oberkampf, W. L., 2011. “A comprehensive framework for verification, validation, and uncertainty quantification in scientific computing", Computer Methods in Applied Mechanics and Engineering, 200: 2131-2144.

[18] Coleman, H. W., Steele, W. G., 2009. Experimentation, Validation, and Uncertainty Analysis for Engineers, 3rd ed., John Wiley \& Sons, Hoboken, New Jersey

[19] Padulo, M., 2009. Computational Engineering Design Under Uncertainty - An aircraft conceptual design perspective, PhD diss., Cranfield University: 127-144.

[20] Modelica 2012. https://modelica.org/.

[21] Dymola 2012. http://www.3ds.com/products/catia/portfolio/ dymola.

[22] Simpson, T., W., et al., 2001, "Metamodels for Computerbased Engineering Design: Survey and Recommendations", Engineering with Computers, 17, 2: 129-150.

[23] Andersson, H., 2012. Variability and Customization of Simulator Products - A Product Line Approach in Model Based Systems Engineering, PhD diss. No. 1427, Linköping University: 33-35.

[24] Persson, J. and Ölvander, J. 2011. "Comparison of Sampling Methods for a Dynamic Pressure Regulator", $49^{\text {th }}$ AIAA Aerospace Sciences Meeting, Orlando, Florida 